\title{
EBOLA VIRUS OUTBREAK THE PRESENT SITUATION IN SOUTH EAST ASIA PERSPECTIVE
}

\author{
Chaudhary $\mathrm{S}^{1}$, Pahwa $\mathrm{VK}^{2}$, Bhatia $\mathrm{BD}^{3}$ \\ 1. Execuitve Editor, \\ 2. Chief Editor, \\ 3. Associate Chief Editor
}

The world is at present experiencing the worst Ebola outbreak in history. World Health Organizationon on 8 August 2014 has declared the Ebola outbreak in West Africa a Public Health Emergency of International Concern. Ebola viruses (genus Ebolavirus, family Filoviridae) cause often fatal, hemorrhagic fever in several species of simian primates including human and have mortality rate up to $90 \%$. Ebola first appeared in 1976 in 2 simultaneous outbreaks, in Nzara, Sudan, and in Yambuku, Democratic Republic of Congo. The latter was in a village situated near the Ebola River, from which the disease takes its name. There are five species of Ebola viruses. The current outbreak is caused by species Zaire Ebolavirus, which was named after Zaire (now Democratic Republic of the Congo), where it was first found. Sudan Ebolavirus, Bundibugia Ebolavirus, Reston Ebolavirus and Taï Forest Ebolavirus are the other species of Ebola virus. Since the discovery, Ebola virus genetically stayed pretty much the same. There's no expectation that it will change significantly in future. Zaire Ebolavirus has had an overall survival rate of roughly 30 percent and has the highest death rate of the five different species of Ebola virus. The Ebola virus has now involved in countries like: Guinea, Sierra Leone, Liberia and Nigeria. Travel associated Ebola cases are also reported from USA, Spain, Senegal and Mali. As of 23 October 2014, 10141 (probable, confirmed and suspected) cases and 4922 deaths have been reported in the current outbreak of Ebola virus disease. In Africa, fruit bats, particularly of the Pteropodidae family Hypsignathus monstrosus, Epomopsfranqueti and Myonycteristorquata, are considered possible natural hosts for Ebola virus. The involvement of other species in the Ebola virus transmission cycle is unclear, especially for domesticated animals. Dogs and pigs are so far the only domestic animals identified as species that can be infected with Ebola virus. In 2009 Reston- Ebolavirus was the first Ebola virus reported to infect swine with indicated transmission to humans. In a survey in Gabon sero-prevalence for Ebola virus in dogs was found over 30\% during the Ebola outbreak in 20012002. Ebola is introduced into the human population through close contact with the blood, secretions, organs or other body fluids of infected animals like chimpanzees, gorillas, fruit bats, monkeys, forest antelope and porcupines found ill or dead or in the rainforest. It is believed that the disease may have spread to humans via "bushmeat" and spread in the human population through human-to-human transmission.

Ebola virus cause an acute febrile illness characterized by multisystem involvement that begins with the abrupt onset of fever headache, sore throat and muscle pain and proceeds to vomiting, diarrhoea, chest pain, prostration, maculo-papular rash and bleeding manifestations. Laboratory findings include low white blood cell and platelet counts and elevated liver enzymes. Ebola virus infections can be diagnosed definitively in a laboratory through several types of tests like antibody-capture enzyme-linked immunosorbent assay (ELISA) which can detect IgM and IgG antibodies, antigen detection tests, serum neutralization test, reverse transcriptase polymerase chain reaction (RT-PCR) assay, electron microscopy and virus isolation by cell culture. No licensed vaccine for Ebola is available. Severely ill patients require intensive supportive care. Patients are frequently dehydrated and require oral rehydration with solutions containing electrolytes or intravenous fluids. No specific treatment is available. New drug therapies are being evaluated.

Ebola spreads by touching the bodily fluids, body of an Ebola patient or touching bedding or other contaminated inanimate objects. After that, virus has to get into the person by, for example, touching food and eating it. Health care personnel doctor, nurses and paramedics are of great risk of getting the infection and disease while attending Ebola patients. Till now many health professionals have developed Ebola and died. Liberian doctor Samuel Brisbane died of Ebola on July 26, 2014. That makes him the first Liberian doctor to die of Ebola in the current outbreak. Sheik Umar Khan was the top doctor fighting against Ebola in Sierra Leone also died from the disease.

Researches in developing Ebola vaccine are making some progress and there are a couple of different vaccine platforms that have shown to be protective with non-human primates. The most notable development is monoclonal antibodies that are engineered to bind with the Ebola virus. They are protective when given right after exposure or a few days after the illness but still large human trials are required. The problem in developing new drug or vaccine, is the economics of drug development. These outbreaks affect the poorest communities on the planet. Although they do create incredible upheaval, they are relatively rare events. So if you look at the interest of pharmaceutical companies, there is not huge enthusiasm to take an Ebola drug through phase one, two, and three of a trial and make an Ebola vaccine that may be a few tens of thousands or hundreds of thousands of people will use. There's not a huge demand for this, and could stand to lose money. There are concerns, for example, about Ebola being used as bioterrorism, and that drives a lot of the funding for this. There is one vaccine that has gone through phase one testing. Now the challenge is how we get into phase two trials, which test efficacy. A Canadian pharmaceutical company called Tekmira has been at work for the past few years on an Ebola treatment called TKM- 
Ebola. It has been among the most advanced attempts at a drug that could protect against, and treat, the disease. TKM-Ebola may not be the only hope another unnamed Ebola vaccine has been added to the FDA's Fast Track, and will be injected into human subjects beginning in September. Although no licensed Ebola virus vaccines are currently available, vaccine research in non-human primates, the 'gold standard' animal model for Ebola virus, has produced several promising candidates. A combination of DNA vaccination and a recombinant adenovirus serotype 5 boost resulted in cross-protective immunity in non-human primates. A recombinant vesicular stomatitis vaccine vector protected nonhuman primates in pre- and post-exposure challenge studies. Several antiviral therapies are currently under investigation, but only a few of these have been tested in non-human primate models. Antisense therapies, in which oligonucleotides inhibit viral replication, have shown promising results in non-human primates following post-exposure treatment. Then in present situation when there are not much treatment option, how to stop the Ebola outbreak.

With improvement in air travel, borders don't mean that much when it comes to disease. Nepal is vulnerable to Ebola epidemic as hundreds of its security officers and personnel are deployed under the UN peacekeeping missions in West African nations. Nepal is also one of the top destinations for foreign tourists. It's entirely possible that at some point, someone infected with Ebola could get on a plane and land in the Nepal during incubation period which is two days to three weeks. Then what? What would happen if the disease came to Nepal? Experts say, at places with good medical facilities, Ebola outbreak can be contained pretty quickly but can be very difficult in resource poor country like ours and it may be a catastrophe. The outbreak in West Africa is so severe for a number of key reasons, including a lack of resources, inadequate infection control measures, and mistrust of health workers. Ebola is not contagious during incubation period but it is contagious once they are symptomatic. Initial presentation either appears as the flu like or traveler's diarrhea. From previous epidemics it has been calculated that 1 primary human case generates only 1 to 3 secondary cases on average, as compared with 14 to 17 for measles in West Africa.These observations point to immediate priorities for control: early diagnosis with patient isolation, contact tracing, strict adherence to biosafety guidelines in laboratories, barrier nursing procedures and use of personal protective equipment by all health care workers, disinfection of contaminated objects and areas, and safe burials. Any suspected case of Ebola would be treated as a potential risk until tests come back negative. This means that standard procedures to protect other patients and health-care workers from the patient's bodily fluids would be put into place. Anything that touches the patient would be sterilized or disposed of in a safe manner and if the patient dies, the body would be carefully handled. The most crucial first step is to identify everyone who's actually been infected with Ebola so that they can be isolated and get medical treatment. Then experts should interview the patient, her relatives, and other potential close contacts to monitor them for 21 days and make sure that they don't spread the disease to others. The World
Health Organization says that "low coverage of contact tracing" is a key problem in the Ebola outbreak in Liberia.

Proper disposal of dead bodies are also very important. The corpses of Ebola patients can still transmit the virus through bodily fluids like sweat. One of the big mysteries in the Ebola outbreak in West Africa is where the virus came from in the first place. In Sierra Leone more than a dozen women became infected after attending the funeral of a traditional healer who had been treating Guinean Ebola patients and contracted the disease. This means that anyone touching a dead body should be using safety precautions such as gloves. Funerals and burials should be conducted in the presence of fully trained personnel so as to reduce the risk of spreading infection. In last we need to do patients education even in countries that have yet to have a single case of the disease. The idea here is to prepare those countries before the outbreak spreads there. The Ministry of Health and Population has also decided to manage treatment of Ebola virus at Bir Hospital and Sukraraj Tropical and Infectious Disease Hospital in Kathmandu. The government has also decided to apply precautionary measures by setting quarantine health desks at the Tribhuvan International Airport (TIA) and border checkpoints. But situation is very poor as there is no proper isolation ward and facility to quarantine and treat Ebola patients. The Epidemiology and Disease Control Division, responsible for responding to an epidemic outbreak, is yet to set up a quarantine system at TIA.The Ministry has also written to the government hospitals above 50bed, and all other hospitals above 200 beds to keep vigil on the suspected cases of the disease.

\section{Further Reading}

1. http://www.msf.org.uk/ebola

2. World Health Organization. Global Alert and Response. (http://www.who.int/csr/disease/ebola/en/)

3. WHO Ebola Response Team. Ebola virus disease in west Africa the first 9 months of the epidemic and forward projections. NEngl JMed 2014. published online Sept 23. DOI:10.1056/ NEJMoa1411100.

4. Galvani AP, Ndeffo-Mbah ML, Wenzel N, Childs JE. Ebola vaccination: if not now, when?. Ann Intern Med 2014. published online Aug 21. DOI:10.7326/M14-1904.

5. World Health Organization. WHO statement on the meeting of the International Health Regulations Emergency Committee regarding the 2014 ebola outbreak in West Africa

(http://www.who.int/mediacentre/news/statements/2014/ebola20140808/en/).

6. Centers for Disease Control and Prevention. Ebola outbreaks 2000-2014. 2014

(http://www.cdc.gov/vhf/ebola/resources/outbreaks.html). 